\title{
FGF Signaling during embryo development regulates cilia length in diverse epithelia
}

\author{
Judith M. Neugebauer, Jeffrey D. Amack ${ }^{\dagger}$, Annita G. Peterson, Brent W. Bisgrove, and H. \\ Joseph Yost \\ Department of Neurobiology \& Anatomy, University of Utah School of Medicine, Eccles Institute \\ of Human Genetics, Bldg. 533, Room 3160, 15 North 2030 East, Salt Lake City, UT 84112-5330
}

\begin{abstract}
Cilia are cell surface organelles found on most epithelia in vertebrates. Specialized groups of cilia play critical roles in embryonic development, including left-right (LR) axis formation. Recently, cilia have been implicated as recipients of cell-cell signaling1, 2. However, little is known about cell-cell signaling pathways that control the length of cilia3. Here we provide several lines of evidence showing that fibroblast growth factor (FGF) signaling regulates cilia length and function in diverse epithelia during zebrafish and Xenopus development. Morpholino (MO) knockdown of FGF receptor 1 (FGFR1) in zebrafish cell-autonomously reduces cilia length in Kupffer's vesicle $(\mathrm{KV})$ and perturbs directional fluid flow required for LR patterning of the embryo. Expression of a dominant-negative FGFR (DN-FGFR), treatment with SU5402, a pharmacological inhibitor of FGF signaling, or genetic and morpholino reduction of redundant FGF ligands FGF8 and FGF24, reproduces this cilia length phenotype. Knockdown of FGFR1 also results in shorter tethering cilia in the otic vesicle and shorter motile cilia in the pronephric ducts. In Xenopus, expression of a DNFGFR results in shorter monocilia in the gastrocoel roof plate (GRP) that control LR patterning4 and in shorter multicilia in external mucociliary epithelium. Together, these results suggest a fundamental and highly conserved role for FGF signaling in the regulation of cilia length in multiple tissues. Abrogation of FGFR1 signaling down-regulates expression of two ciliogenic transcription factors, foxj 1 and $r f x 2$, and the intraflagellar transport (IFT) gene, polaris, suggesting that FGF signaling mediates cilia length through an FGF8/FGF24 - FGFR1- IFT pathway. We propose that a subset of developmental defects and diseases ascribed to FGF signaling are due in part to loss of cilia function.
\end{abstract}

FGF ligands bind and activate cell surface FGF receptors (FGFR) to mediate multiple processes during embryogenesis. One ligand, FGF8, has been proposed to play divergent roles in LR patterning5-9; as a left determinant in mouse and a right determinant in chick and rabbit. Experimental manipulations of FGFR function allow cell-autonomous alterations of FGF signaling not possible with manipulations of multiple secreted ligands that activate a given receptor. Using this approach, we investigated the roles of FGFR1 in zebrafish

Users may view, print, copy, and download text and data-mine the content in such documents, for the purposes of academic research, subject always to the full Conditions of use:http://www.nature.com/authors/editorial_policies/license.html\#terms

Correspondence and requests should be addressed to HJY (jyost@genetics.utah.edu)..

†Present address: Department of Cell and Developmental Biology, SUNY Upstate Medical University 750, E. Adams St., Syracuse, NY 13210 
development. To elucidate the role of FGFR1 signaling in LR development, we analyzed the expression of southpaw (spaw; zebrafish nodal homolog) the earliest known asymmetrically expressed gene10. Knockdown of FGFR1 with two distinct antisense morpholinos (MO) perturbed the normal left-sided expression of spaw in lateral plate mesoderm (LPM) (Fig. 1a-c). Ets transcription factors pea3 and erm, downstream targets of FGF signaling11, were down-regulated in $f g f r l$ morphants (Supplemental Fig. 1), indicating the efficacy of MO knockdown. Markers of notochord (no tail, leftyl, sonic hedgehog)12, 13 and floorplate (sonic hedgehog), were found to be normal in $f g f r l$ morphants (Supplemental Fig. 2), suggesting the barrier role of the embryonic midline is intact. These results indicate FGFR1 signaling is required early in LR development, preceding asymmetric expression of spaw.

spaw asymmetry is dependent on Kupfer's vesicle (KV), a ciliated epithelium structure that creates directional fluid flow12-14, analogous to 'nodal flow' in mouse15. Fgfrl mRNA is expressed in KV and surrounding tailbud (Fig. 1d, e). To determine whether FGF signaling functions cell-autonomously in KV cells to control spaw asymmetry, we generated chimeric $\mathrm{DFC}^{f g f r l} \mathrm{MO}$ embryos in which $f g f r l$ is knocked-down in DFC/KV (dorsal forerunner cells; $\mathrm{KV}$ precursor cells) lineages 12 but not the rest of the embryo. Similar to embryo-wide knockdown of $f g f r l$, DFC $f g f r l \mathrm{MO}$ embryos had significant alterations in spaw expression relative to DFC Control MO (p<1.19e-05; Fig. 1c, f-h). As an important control, the effects of knockdown of FGFR1 in yolk alone (yolk ${ }^{f g f r l ~ M O}$ ) were similar to yolk ${ }^{\text {control MO }}$ (Fig. 1h; $\mathrm{p}<0.90)$. These results indicate that cell-autonomous FGFR1 signaling in DFC/KV cells is necessary for asymmetric expression of spaw in LPM.

What role does FGFR1 signaling play in DFC/KV function? Atypical Protein Kinase C (aPKC), an apical marker of polarized KV epithelial cells16, revealed that KV were of normal size and shape in $f g f r l$ morphants (Fig. 2a, b; $\mathrm{n}=15 / 15$, control $\mathrm{n}=16 / 16$ ), in contrast to dismorphic KV phenotypes seen in $n t l$ or spt mutants and morphants16. Thus, morphogenesis of the KV epithelium is not dependent on FGFR1 signaling. However, KV cilia were shorter in $f g f r l$ MO-1 compared to Control morphants and WT embryos (Fig. 2ac; $\mathrm{p}<1.9 \mathrm{e}-08$ ); the number of cilia was unaltered (Fig. 2; p<0.98). Similar results were obtained from fgfrl MO-2 (data not shown). Importantly, Xenopus fgfrl mRNA17 rescued cilia defects induced by fgfrl MO (Fig. 2c, p<4.70e-05), demonstrating that cilia defects in fgfrl morphants are specific to FGFR1 knockdown.

Additional approaches were used to assess the requirement of FGFR signaling for normal KV cilia length. Embryos treated during shield stage with a pharmacological inhibitor of FGFR activity, SU540218, 19, had shorter cilia compared to DMSO-treated controls (Fig. $2 \mathrm{~d} ; \mathrm{p}<3.26 \mathrm{e}-06$ ). Treatment at subsequent stages altered LR development but not cilia length (JMN \& HJY, in prep), suggesting FGF signaling plays multiple stage-specific roles in LR development. We analyzed transgenic embryos carrying a heat-shock inducible dominant negative FGFR1 (DN-FGFR) fused to eGFP which identifies transgenic embryos from their non-transgenic siblings 20 . When DN-FGFR was activated at $60 \%$ epiboly, transgenic embryos had shorter cilia compared to heat-shocked non-transgenic siblings (Fig. 2e; $\mathrm{p}<6.94 \mathrm{e}-03$ ) and non-heat-shocked siblings (Fig. 2e; p<6.99e-03), both of which had normal length cilia (Fig. 2e; $\mathrm{p}<0.61$ ). Brief hyperactivation of FGF signaling by inducible FGFR21 avoided overexpression defects but did not increase cilia length (Supplemental Fig 3). 
Which ligands signal through FGFR1 to control cilia length? FGF8 binds several FGFRs22 and FGFR1 morphants phenocopy midbrain-hindbrain defects seen in zebrafish FGF8 (acerebellar; ace) mutants5, 23. This suggests FGFR1 is a functional receptor for FGF823. ace mutants have LR defects and a minority fail to form a KV lumen5. We found $f g f 8$ deficient embryos express KV differentiation markers ( $\operatorname{sox} 17$, $\mathrm{n}=87 / 98$ ), form an epithelium with normal apical-basal polarity ( $\mathrm{aPKC}, \mathrm{n}=10 / 10)$, and despite $33 \%$ not filling the $\mathrm{KV}$ lumen, develop normal numbers of cilia with normal length (Fig. 2f; $\mathrm{p}<0.53$ ).

Another FGF ligand, $f g f 24$, has overlapping expression with $f g f 8$ in and around DFC/KV cells24. fgf 24 mutants (ikarus; ika) 25 and siblings had normal length KV cilia (average cilia length $=6.2 \mu \mathrm{m} ; 498$ cilia; 12 embryos). To test for redundant function of FGF8 and FGF24, we injected $f g f 24 \mathrm{MO}$ into ace mutants to reduce the amount of FGF8/FGF24 activity. ace heterozygotes injected with $f g f 24 \mathrm{MO}$ had shorter $\mathrm{KV}$ cilia than uninjected ace heterozygotes (Fig. 2f; p<0.015), and ace homozygotes injected with $f g f 24 \mathrm{MO}$ had KV cilia lengths comparable to $f g f r 1$ morphants (Fig.2f; p<3.63e-07). Similarly, ika mutants injected with $f g f 8 \mathrm{MO}$ had shorter cilia (Supplemental Figure 4). WT, ika mutants and siblings injected with $f g f 24 \mathrm{MO}$ had normal length cilia (Fig. 2f; p $<0.28$ ), arguing against off-target MO effects. These results indicate that FGF8 and FGF24 ligands function, likely through FGFR1, to control cilia length. Thus, results from MO against FGFR1, pharmacological inhibitors of FGFRs, transgenic expression of DN-FGFR, and mutants and MO of multiple FGF ligands indicate that FGF signaling is necessary to control KV cilia length.

To assess whether cilia-driven directional fluid flow in KV was altered by the cilia defects in fgfrl morphants, we tracked movement of fluorescent beads injected into the lumen of KV13. In control morphants, fluorescent beads exhibited a persistent counter-clockwise directional flow (Fig. 2i, Supplemental Movie 1). In contrast, beads in fgfrl morphants had no persistent directional flow (Fig. 2j, Supplemental Movie 2) indicating FGF signaling controls LR patterning by regulating cilia length and KV fluid flow prior to initiation of asymmetric spaw expression.

The discovery that FGF signaling plays a role in LR patterning by regulating cilia suggests other developmental roles attributed to FGF signaling might be due to cilia defects. To determine whether FGF-dependent regulation of cilia length is a more general developmental mechanism, we examined cilia in two epithelia that express FGFR1, the pronephric ducts and ear (otic vesicle; Supplemental Fig. 5b, c). Pronephric ducts are primitive excretory organs containing motile cilia14. Inhibition of FGF signaling during Xenopus embryogenesis inhibits pronephric development26, but no mechanism has been elucidated. Pronephric duct cilia at 26 somite stage (SS) were shorter in $f g f r l$ morphants compared to WT embryos (Fig. 3a, b, e; p<4.24e-04). Consistent with pronephric cilia defects, $f g f r 1$ morphants develop cystic kidneys (Supplemental Fig. 6). In the zebrafish ear, two types of cilia are required for otolith formation: tethering cilia and motile cilia. Tethering cilia attract seeding granules and when reduced in number or length, granules are not organized correctly for otolith formation19. In zebrafish FGF8 or FGFR1 knock-down perturbs otic vesicle and otolith formation23, and otic vesicle cilia number is altered when FGF signaling is pharmacologically inhibited18. Here, $f g f r l$ morphants had shorter tethering cilia and otolith defects (Fig. 3c-d, d, Supplementary Fig. 6d-e; p< 1.1e-07), suggesting the 
otic vesicle and otolith defects seen in $f g f r l \mathrm{MO}$ are due to defects in cilia length. Thus FGF signaling controls cilia length and function in multiple tissues during zebrafish development.

To explore whether control of cilia length by FGF signaling is conserved in vertebrates, two types of epithelial cilia were examined in Xenopus laevis: monocilia on gastrocoel roof plate (GRP) implicated in LR patterning4, and mucociliary epithelial cilia that move fluid across the external epidermis3. Since DN-FGFR causes gastrulation defects when expressed ubiquitously during early embryogenesis, we co-injected DN-FGFR and GFP mRNA into cell lineages that contribute to either the GRP or mucociliary epithelium (Supplemental Fig. 1d-f). GRP cells co-expressing GFP and DN-FGFR had shorter cilia compared to neighboring GRP cells in the same embryo (p<6.0e-03; Fig. 3i-j, m) and GRP cells in embryos expressing GFP alone (p<2.7e-03; Fig. 3g-h, m). In mucociliary epithelial, cells coexpressing GFP and DN-FGFR had shorter cilia compared to cells expressing GFP alone ( $\mathrm{p}<0.019$; Fig. 3k-i, n). These results indicate that FGF signaling controls cilia length in diverse epithelia, and suggests that the regulation of cilia length by FGF signaling is evolutionarily conserved.

How does FGFR1 regulate cilia length? To address this, we analyzed cell differentiation, epithelial cell polarization and cilia formation of $\mathrm{KV}$ cells in zebrafish16. In $\mathrm{fg} f \mathrm{fr}$ morphants, two markers of the DFC/KV cell lineage, sox1712 and dnah913, showed similar expression in WT and $f g f r l$ morphants, indicating correct DFC/KV cell differentiation (Fig. 4a-d, i). Apical membrane marker aPKC and tight junction marker ZO-1 revealed apicalbasal polarity in KV cells was intact in $f g f r l$ morphants compared to WT controls (Supplemental Fig. 7a-d). Further, cilia in $f g f r l$ morphants were correctly positioned at the apical surface facing the KV lumen (Supplemental Fig. 7e, f). In contrast to the apparent normal differentiation and polarization of $\mathrm{KV}$ cells in $f g f r l$ morphants, two members of transcription factor families implicated in ciliogenesis27, 28, foxJI and $r f x 2$ (BWB and HJY, in preparation) were down-regulated in these embryos (Fig. 4e, f, i). Correspondingly, expression of polaris, an intraflagellar transport gene (Ift88) required for normal length cilia in zebrafish29, was diminished in $f g f r l$ morphants (Fig. 4g-i). Reduced polaris expression is consistent with IFT-defective phenotypes seen in $f g f r 1$ morphants, including curved body axis, kidney cysts and shortened cilia (Fig. 2a-f, Supplemental Fig. 6). From these results, we propose that FGF8 and FGF24 activate FGFR1 cell-autonomously in KV cells to maintain a transcriptional network that allows normal expression of IFT proteins required for normal length cilia (Fig. 4j).

Monocilia are found on almost all cells and have been implicated as sites for receiving or modulating cell-cell signaling pathways such as Hedgehog1, PDGF1 and Wnt2. Interactions among signaling pathways are of great interest in understanding how cells integrate diverse signals. Extrapolating from our discovery of a link between FGF signaling and cilia function in zebrafish and Xenopus, we propose that (1) some of the apparent interactions between FGF signaling and other cell signaling pathways might be due to FGF-dependent changes in cilia, which then influence the ability of cells to receive and integrate other cell-cell signals, and (2) a spectrum of developmental defects and human diseases due to defects in FGF signaling might be due to defects in cilia length or function. 


\section{Methods Summary \\ Xenopus mRNA Injections}

For Xenopus GRP monocilia analysis, embryos were injected with 200 pg GFP mRNA alone (lineage tracer) or co-injected with 400 pg DN-FGFR mRNA into two dorsal cells of 32-cell embryo. For Xenopus epithelial cell analysis, embryos were injected with $200 \mathrm{pg}$ GFP mRNA alone or co-injected with 600 pg dnFGFR mRNA into a single ventral cell of a 16-cell embryo.

\section{Statistics}

Cilia measurements were analyzed using a two-tailed student's T-test, and analysis of spaw proportions were conducted using Fisher's exact test. In a given embryo each cilium was measured in the tissue of interest and the average cilia length per embryo was determined. Averages for controls and experimentals were compared within each clutch of embryos. Outcomes were the same using a second analytical approach in which all cilia lengths were pooled and compared across all series of experiments. Analysis was done by R-Commander software package within the R Statistical Software platform30. Results are considered significant when $\mathrm{p}<0.05$ and results are expressed as mean \pm standard error of the mean.

\section{Supplementary Material}

Refer to Web version on PubMed Central for supplementary material.

\section{Acknowledgements}

We thank A. Moon and M. Condic for critical discussions on the manuscript; M. Karthikeyan, J. Shen, D. Coombs, and E. Martini for technical help, S. Miyagawa-Tomita, K. Poss, and H. Issacs for reagents. This work was supported by American Heart Association predoctoral fellowship to JMN, NRSA Postdoctoral fellowship to JDA and grants from NHLBI, NICHD, and Primary Children's Medical Foundation to HJY.

\section{Appendix}

\section{Methods}

\section{Zebrafish and Xenopus embryo culture}

Oregon AB WT zebrafish (Danio rerio) were collected from natural matings, and were injected, raised and staged as described previously13. Heterozygote crosses with ace ${ }^{\text {ti282a }}$, $f g f 24^{t 22030}$, and hsp70:dn-fgfr1were used to produce ace and $f g f 24$ mutant embryos and hsp:dn-fgfr1 transgenic embryos respectively5, 20, 23, 25. Hsp70:dnfgfr1 embryos from heterozygote crosses were incubated at $28^{\circ} \mathrm{C}$ (no heat-shock activation) or at $60 \%$ epiboly for one hour at $37{ }^{\circ} \mathrm{C}$ (heat-shock activation) and then returned to $28^{\circ} \mathrm{C}$ until collected for IHC. Xenopus embryos were obtained using standard methods as previously described3.

\section{Morpholino and mRNA injections}

Antisense morpholino oligonucleotides (MO) were obtained from Gene Tools, LLC and Open Biosystems. Fluorescently labeled MOs against FGFR1 were designed using previously described sequences: translation blocking 3-carboxyfluorescein-labeled $f g f r l$ 
MO-1 (5'- GCAGCAGCGTGGTCTTCATTATCAT-3')23, 31, translation blocking 3carboxyfluorescein-labeled $f g f r l$ MO-2 (5'-CAAAGATCCTCTACATCTGAACTCC-3')31. The $f g f 24$ MO (5'-AGGAGACTCCCGTACCGTACTTGCC-3') and the 3-lissamine-labeled fgf8 MO (5'-TAGGATGCTCTTACCATGAACGTCG-3') have also been previously described24, 32. Fluorescein-labeled standard negative control (5'CCTCTTACCTCAGTTACAATTTATA-3') from Gene Tools, LLC was used in control injections. MO was injected into 1-4 cell zebrafish embryos for whole embryo protein knock-down experiments 13 . A volume of $1 \mathrm{nl}$ was delivered containing $5 \mathrm{ng}$ of $f g f 24 \mathrm{MO}$, $4 \mathrm{ng}$ of $f g f r l \mathrm{MO}-1,8 \mathrm{ng}$ of $f g f r l \mathrm{MO}-2$, or $4 \mathrm{ng}$ of Control MO. For DFC ${ }^{\mathrm{MO}}$ experiments, fluorescent $\mathrm{MO}$ was injected into the yolk of embryos at 500-1000 cell stage and embryos were selected by fluorescent microscopy for MO accumulation in DFC as previously described12. To control for activity of the protein of interest in the yolk alone we used yolk ${ }^{\mathrm{MO}}$ control injections: fluorescent $\mathrm{MO}$ was injected into dome-30\% epiboly embryos, and embryos were selected by fluorescent microscopy for MO diffusion throughout the yolk. For $\mathrm{DFC}^{\mathrm{MO}}$ and yolk $\mathrm{MO}^{\mathrm{MO}}$ injections $1 \mathrm{nl}$ was delivered containing $2 \mathrm{ng}$ of $f g f r l \mathrm{MO}-1$ or $2 \mathrm{ng}$ of Control MO. Capped xFGFR1, DN-FGFR, iFGFR21 and GFP mRNAs were made from linearized plasmid using the using the mMessage machine SP6 transcription kit (Ambion)17. For MO rescue experiments, 100 pg of xFGFR1 was injected alone or coinjected with 5 ng of $f g f r l$ MO-1 into 1-4 cell stage zebrafish embryos. For iFGFR experiments, $2.5 \mathrm{pg}$ of iFGFR was injected into 1-4 cell stage zebrafish embryos.

\section{In Situ Hybridization}

Digoxigenin RNA probes were generated using a Roche DIG RNA labeling kit. cDNA templates used include spaw10, shh13, ntl12, fgfr123, sox1712, pea311, erm11, lefty 113, dnah913, polaris29, foxJ1 (BWB unpublished), $r f x 2$ (BWB unpublished). In situ hybridization were performed as previously described13, with automated wash and antibody incubation using a Biolane HTI machine (Huller and Huttner HG). After post-fixation, embryos were cleared in 100\% EtOH for imaging. Embryos were stored in $70 \%$ glycerol and images were obtained and processed using a Nikon Coolpix5000 camera and Photoshop Software (Adobe).

\section{Immunofluorescence Microscopy}

For zebrafish immunohistochemistry, embryos were fixed in $4 \%$ paraformaldehyde at $4{ }^{\circ} \mathrm{C}$, dehydrated in a $\mathrm{MeOH}$ series, stored in $100 \% \mathrm{MeOH}$, rehydrated, boiled in $1 \mathrm{mM}$ EDTA for five minutes (except IHC for pronephric cilia), and subsequently blocked for 1 hour in PBS containing 5\% sheep serum, $1 \%$ BSA, $1 \%$ DMSO, and 0.1\% Triton-X. Embryos were incubated in primary antibody including mouse anti- acetylated Tubulin (1:300, Sigma T-6793), rabbit anti-atypical Protein Kinase C $\zeta$ (1:100; Santa Cruz sc-216), and mouse antiZO-1 (1:150; Zymed 33-9100). After washes with PBS/0.1\% Triton-X/1\% DMSO/1\% BSA embryos were blocked for 1 hour and incubated in secondary antibody, including goat antirabbit Alexa Fluor 647 and goat anti-mouse Alexa Fluor 488. Embryos were cleared and mounted in Slow Fade Reagent (Molecular Probes). Images were acquired using an Olympus Fluoview FV300 laser scanning confocal microscope and assembled using ImageJ (NIH) and Photoshop (Adobe) software. Confocal z-series images were assembled to present the sum of the focal planes; cilia length was measured using Metamorph software 
(Universal Imaging Corp). For GRP monocilia imaging, injected Xenopus embryos were collected at stage 1733, the vitelline membrane removed, fixed overnight in 4\% PFA in PBS, dehydrated in methanol and stored at -20C. Embryos were dissected following rehydration to expose GRP cilia according previous methods4. For epithelial cilia analysis, injected embryos were collected at stage 26 and kept whole3. Embryos were blocked in 10\% lamb serum in PBS/0.1\% Triton-X (PBST), with PBST only washes. Cilia were labeled as for zebrafish and injected cells were visualized using a polyclonal GFP antibody (1:400; Torrey Pines Biolabs). Anti-mouse Alexa fluor 568 and anti-rabbit Alexa fluor 488 secondary antibodies were used. Samples were mounted in PBST and imaged using an Olympus Fluoview FV300 confocal microscope. To measure epithelial cilia length, images were processed using Fluoview software to render the cilia in the $\mathrm{x}-\mathrm{z}$ plane and then images and cilia length for both epithelial and GRP cilia were measured as for zebrafish.

\section{KV Flow Analysis}

Embryos were dechorionated at 6-8 SS and mounted in 1\% low melt agarose. Fluorescent beads (0.5-2 $\mu \mathrm{m}$; Polysciences, Inc.) were injected into KV and imaged on a Leica DMRA compound microscope using a 40x Plan Apo objective using a Coolsnap HQ digital camera (Photometrics), Metamorph (Universal Imaging Corp) to track individual beads and calculate velocity and Quicktime (Apple) to display movies.

\section{Pharmacological Treatments}

Shield-stage embryos were incubated in 24 well tissue culture dishes (25-30 embryos per well) in either SU5402 (Calbiochem)18, 19 resuspended in DMSO or AP20187 (Ariad) resuspended in EtOH, diluted into embryo water to a concentration of $20-25 \mu \mathrm{M}$ for SU5402 (concentration dependant on drug lot) or $1.25 \mu \mathrm{M}$ for AP20187. For a vehicle control, an equivalent volume of DMSO or EtOH was added to embryo water. At after 1 hour, embryos were washed with embryo water and incubated in the 24 well dishes until fixed for IHC.

\section{References}

31. Thummel R, et al. Inhibition of zebrafish fin regeneration using in vivo electroporation of morpholinos against fgfr1 and msxb. Dev Dyn. 2006; 235:336-346. [PubMed: 16273523]

32. Draper BW, Morcos PA, Kimmel CB. Inhibition of zebrafish fgf8 pre-mRNA splicing with morpholino oligos: a quantifiable method for gene knockdown. Genesis. 2001; 30:154-156. [PubMed: 11477696]

33. Nieuwkoop, PD.; Faber, J. Normal table of Xenopus laevis (Daudin) : a systematical and chronological survey of the development from the fertilized egg till the end of metamorphosis. Garland Pub.; New York: 1994.

\section{References}

1. Eggenschwiler JT, Anderson KV. Cilia and developmental signaling. Annu Rev Cell Dev Biol. 2007; 23:345-373. [PubMed: 17506691]

2. Gerdes JM, et al. Disruption of the basal body compromises proteasomal function and perturbs intracellular Wnt response. Nat Genet. 2007; 39:1350-1360. [PubMed: 17906624]

3. Park TJ, Mitchell BJ, Abitua PB, Kintner C, Wallingford JB. Dishevelled controls apical docking and planar polarization of basal bodies in ciliated epithelial cells. Nat Genet. 2008; 40:871-879. [PubMed: 18552847] 
4. Schweickert A, et al. Cilia-driven leftward flow determines laterality in Xenopus. Curr Biol. 2007; 17:60-66. [PubMed: 17208188]

5. Albertson RC, Yelick PC. Roles for fgf8 signaling in left-right patterning of the visceral organs and craniofacial skeleton. Developmental biology. 2005; 283:310-321. [PubMed: 15932752]

6. Boettger T, Wittler L, Kessel M. FGF8 functions in the specification of the right body side of the chick. Curr Biol. 1999; 9:277-280. [PubMed: 10074453]

7. Fischer A, Viebahn C, Blum M. FGF8 acts as a right determinant during establishment of the leftright axis in the rabbit. Curr Biol. 2002; 12:1807-1816. [PubMed: 12419180]

8. Meyers EN, Martin GR. Differences in left-right axis pathways in mouse and chick: functions of FGF8 and SHH. Science. 1999; 285:403-406. [PubMed: 10411502]

9. Tanaka Y, Okada Y, Hirokawa N. FGF-induced vesicular release of Sonic hedgehog and retinoic acid in leftward nodal flow is critical for left-right determination. Nature. 2005; 435:172-177. [PubMed: 15889083]

10. Long S, Ahmad N, Rebagliati M. The zebrafish nodal-related gene southpaw is required for visceral and diencephalic left-right asymmetry. Development. 2003; 130:2303-2316. [PubMed: 12702646]

11. Roehl H, Nusslein-Volhard C. Zebrafish pea3 and erm are general targets of FGF8 signaling. Curr Biol. 2001; 11:503-507. [PubMed: 11413000]

12. Amack JD, Yost HJ. The T box transcription factor no tail in ciliated cells controls zebrafish leftright asymmetry. Curr Biol. 2004; 14:685-690. [PubMed: 15084283]

13. Essner JJ, Amack JD, Nyholm MK, Harris EB, Yost HJ. Kupffer's vesicle is a ciliated organ of asymmetry in the zebrafish embryo that initiates left-right development of the brain, heart and gut. Development. 2005; 132:1247-1260. [PubMed: 15716348]

14. Kramer-Zucker AG, et al. Cilia-driven fluid flow in the zebrafish pronephros, brain and Kupffer's vesicle is required for normal organogenesis. Development. 2005; 132:1907-1921. [PubMed: 15790966]

15. Nonaka S, et al. Randomization of left-right asymmetry due to loss of nodal cilia generating leftward flow of extraembryonic fluid in mice lacking KIF3B motor protein. Cell. 1998; 95:829837. [PubMed: 9865700]

16. Amack JD, Wang X, Yost HJ. Two T-box genes play independent and cooperative roles to regulate morphogenesis of ciliated Kupffer's vesicle in zebrafish. Developmental biology. 2007; 310:196210. [PubMed: 17765888]

17. Amaya E, Musci TJ, Kirschner MW. Expression of a dominant negative mutant of the FGF receptor disrupts mesoderm formation in Xenopus embryos. Cell. 1991; 66:257-270. [PubMed: 1649700]

18. Millimaki BB, Sweet EM, Dhason MS, Riley BB. Zebrafish atoh1 genes: classic proneural activity in the inner ear and regulation by Fgf and Notch. Development. 2007; 134:295-305. [PubMed: 17166920]

19. Riley BB, Zhu C, Janetopoulos C, Aufderheide KJ. A critical period of ear development controlled by distinct populations of ciliated cells in the zebrafish. Developmental biology. 1997; 191:191201. [PubMed: 9398434]

20. Lee Y, Grill S, Sanchez A, Murphy-Ryan M, Poss KD. Fgf signaling instructs position-dependent growth rate during zebrafish fin regeneration. Development. 2005; 132:5173-5183. [PubMed: 16251209]

21. Pownall ME, et al. An inducible system for the study of FGF signalling in early amphibian development. Developmental biology. 2003; 256:89-99. [PubMed: 12654294]

22. Zhang X, et al. Receptor specificity of the fibroblast growth factor family. The complete mammalian FGF family. J Biol Chem. 2006; 281:15694-15700. [PubMed: 16597617]

23. Scholpp S, Groth C, Lohs C, Lardelli M, Brand M. Zebrafish fgfr1 is a member of the fgf8 synexpression group and is required for fgf8 signalling at the midbrain-hindbrain boundary. Dev Genes Evol. 2004; 214:285-295. [PubMed: 15221377]

24. Draper BW, Stock DW, Kimmel CB. Zebrafish fgf24 functions with fgf8 to promote posterior mesodermal development. Development. 2003; 130:4639-4654. [PubMed: 12925590] 
25. Fischer S, Draper BW, Neumann CJ. The zebrafish fgf24 mutant identifies an additional level of Fgf signaling involved in vertebrate forelimb initiation. Development. 2003; 130:3515-3524. [PubMed: 12810598]

26. Urban AE, et al. FGF is essential for both condensation and mesenchymal-epithelial transition stages of pronephric kidney tubule development. Developmental biology. 2006; 297:103-117. [PubMed: 16872594]

27. Brody SL, Yan XH, Wuerffel MK, Song SK, Shapiro SD. Ciliogenesis and left-right axis defects in forkhead factor HFH-4-null mice. Am J Respir Cell Mol Biol. 2000; 23:45-51. [PubMed: 10873152]

28. Bonnafe E, et al. The transcription factor RFX3 directs nodal cilium development and left-right asymmetry specification. Mol Cell Biol. 2004; 24:4417-4427. [PubMed: 15121860]

29. Bisgrove BW, Snarr BS, Emrazian A, Yost HJ. Polaris and Polycystin-2 in dorsal forerunner cells and Kupffer's vesicle are required for specification of the zebrafish left-right axis. Developmental biology. 2005; 287:274-288. [PubMed: 16216239]

30. Team, R.D.C.. R Foundation for Statistical ComputingVienna. Austria: 2007. 

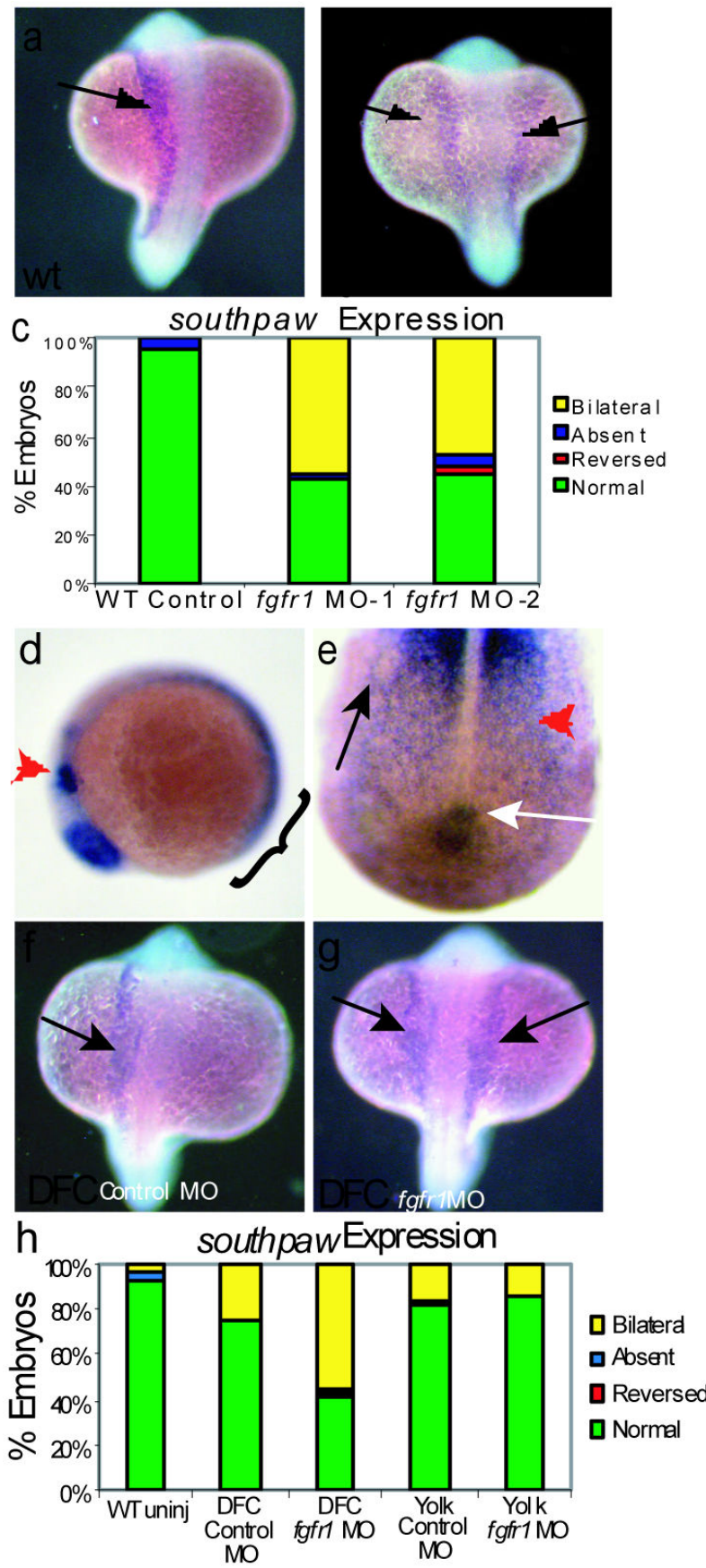

Figure 1. Cell autonomous FGF signaling in Kupffer's Vesicle controls Left-Right patterning (a-b) Dorsal view of left-sided spaw expression (arrow) in WT, and bilateral expression in fgfrl MO 18-20 SS embryos. (c) Percentages of normal (left-sided), reversed, bilateral and absent spaw in WT (n=99), fgfrl MO (n=117) and fgfrl MO2 (n=120). (d-e) fgfrl expression in WT 6 SS embryos. (d) Lateral view (anterior-left) showing $f g f r l$ expression in KV (bracket) and midbrain-hindbrain (red arrowhead). (e) Tailbud showing fgfrl expression in KV (white arrow, dorsal view), presomitic mesoderm (red arrowhead) and lateral plate mesoderm (black arrow). (f-g) spaw expression (arrows) in DFC Control MO and DFCfgfrl MO 
at 18-20 SS. (h) Percentages of spaw expression in DFC and Yolk MO injected embryos. spaw was altered in $\mathrm{DFC}^{f g f r l} \mathrm{MO}(\mathrm{n}=69)$ versus DFC Control MO $(\mathrm{p}<1.19 \mathrm{e}-05 ; \mathrm{n}=121)$, with no difference between Yolk ${ }^{\text {Control MO }}(\mathrm{n}=57)$ and Yolk ${ }^{f g f r l ~ M O}(\mathrm{p}<0.90 ; \mathrm{n}=59)$. 

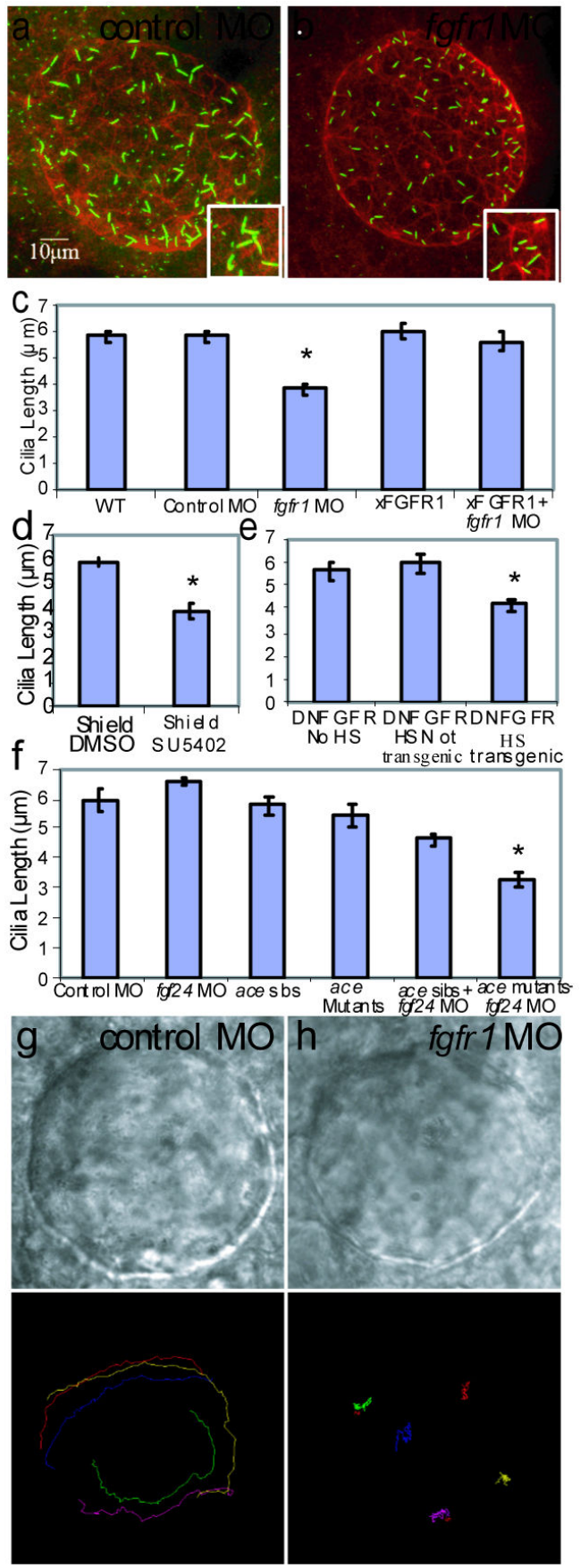

Figure 2. FGF signaling controls cilia length and directional fluid flow in Kupffer's Vesicle (a-b) Confocal images of $10 \mathrm{SS}$ embryos, KV labeled with antibodies against aPKC (red) and acetylated tubulin (green). Control and $f g f r l$ morphants had similar KV structure, but cilia were shorter in $f g f r l$ morphants (compare insets in a and b). (c) Cilia lengths were significantly different ( $\mathrm{p}<2.88 \mathrm{e}-06)$ in $f g f r l$ morphants (688 cilia; 18 embryos) versus Control morphants (437 cilia; 9 embryos). Cilia length was similar in WT uninjected (533 cilia; 10 embryos) and Control morphant ( $\mathrm{p}<0.93$ ), cilia numbers per KV were similar in Control and $f g f r l$ morphants $(\mathrm{p}<0.26)$. Cilia length defects in $f g f r l$ morphants were rescued 
by Xenopus FGFR1 (xFGFR1) mRNA ( $\mathrm{p}<4.70 \mathrm{e}-05 ; 807$ cilia; 21 embryos). Injection of xFGFR1 mRNA alone had no affect on cilia length ( $\mathrm{p}<0.73 ; 526$ cilia, 14 embryos). (d) Embryos treated with SU5402 during shield stage (248 cilia; 12 embryos) had shorter cilia compared to DMSO control embryos ( $\mathrm{p}<3.26 \mathrm{e}-06$; 686 cilia; 15 embryos). (e) Cilia were shorter in transgenic DN-FGFR embryos that were heat shocked at $60 \%$ epiboly (656 cilia; 19 embryos) compared to heat shocked non-transgenic siblings ( $\mathrm{p}<6.94 \mathrm{e}-03 ; 375$ cilia; 10 embryos) and non-heat-shocked siblings ( $\mathrm{p}<6.99 \mathrm{e}-03 ; 910$ cilia; 16 embryos). (f) There was no difference in cilia length $(\mathrm{p}<0.28)$ in $f g f 24$ morphants $(455$ cilia; 10 embryos) versus Control morphants (481 cilia; 10 embryos). However, cilia were shorter when both FGF8 and FGF24 ligands were diminished ( $f g f 24 \mathrm{MO}$ in ace mutants; 12 embryos; 244 cilia), compared to single ligand knockdown (FGF8/ace mutants: $\mathrm{p}<1.39 \mathrm{e}-04 ; 10$ embryos; 480 cilia; fgf $24 \mathrm{MO}$ in ace sibs: $\mathrm{p}<3.44 \mathrm{e}-04 ; 15$ embryos; 643 cilia) and WT ace siblings ( $\mathrm{p}<3.63 \mathrm{e}-07$; 13 embryos; 626 cilia). (g-h) DIC images of bead-injected KVs in Control and fgfrl morphants injected with fluorescent beads. (i-j) Bead paths tracked by Metamorph software. Directional KV fluid flow was absent in $f g f r l$ morphants $(\mathrm{j} ; \mathrm{p}<6.4 \mathrm{e}-15 ; 44$ beads, 9 embryos) compared to counterclockwise flow in Control morphants (i; 39 beads, 8 embryos). Error bars are standard error of the mean (s.e.m). 

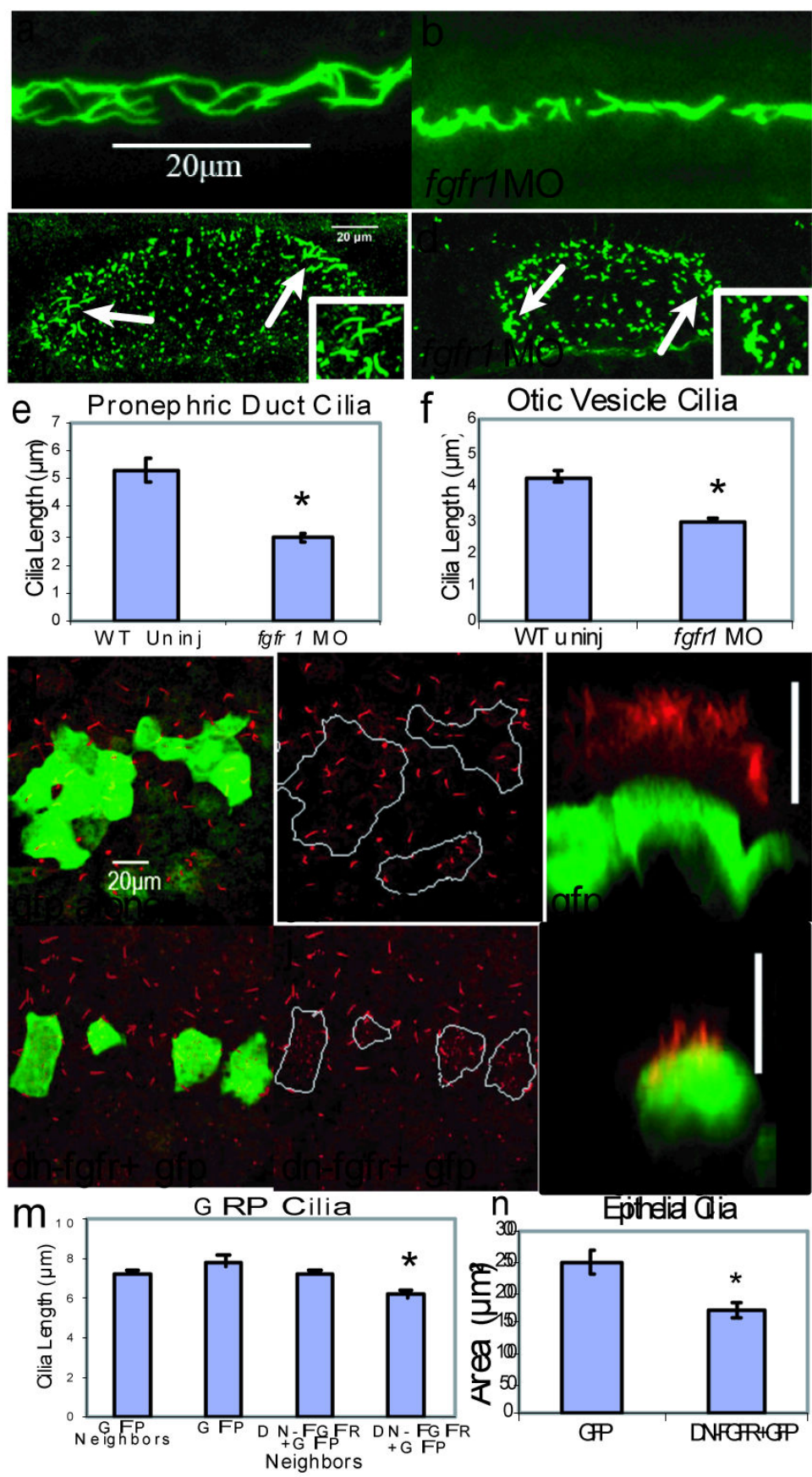

Figure 3. Cilia length in pronephric ducts, otic vesicles, gastrocoel roof plate epithelia and mucociliary epithelia is controlled by FGF signaling

(a-b) Pronephric duct cilia were shorter and disorganized in $f g f r l$ morphants $\left(\mathrm{p}<4.24 \times 10^{-4}\right.$; 528 cilia; 10 embryos,) compared to WT (517 cilia; 10 embryos) 26 SS embryos. (c-d) Otic vesicle tethering cilia (arrows, and inset) were shorter ( $\mathrm{p}<1.10 \mathrm{e}-07)$ in $f g f r l$ morphants $(325$ cilia; 10 embryos) compared to WT embryos (322 cilia; 8 embryos) at 24 hpf. (g-j, m). GRP cilia in Xenopus embryos were normal length in cells expressing GFP alone (green cells in $\mathrm{g}$, outlined in h; 316 cilia 18 embryos, $\mathrm{p}<0.11$ ), neighboring cells (outside boundaries in h; 
653, 18 embryos), and cells neighboring DN-FGFR+GFP expression (outside boundaries in j; 652 cilia 15 embryos, $\mathrm{p}<0.99$ ). In contrast, GRP cilia were shorter in cells expressing DNFGFR+GFP ( $i$, inside boundaries in j; 155 cilia, 15 embryos) compared to neighboring cells $(\mathrm{p}<6.1 \mathrm{e}-03)$ and cells expressing GFP alone $(\mathrm{p}<2.7 \mathrm{e}-03)$. $(\mathrm{k}, \mathrm{l}) \mathrm{Z}$ plane rendering of mucociliary epithelia (scale bar $20 \mathrm{um}$ ), showing shorter cilia in cells expressing DN-FGFR +GFP (13 cells, 7 embryos) compared to controls expressing GFP alone (14 cells, 4 embryos). (n) Multicilia area is reduced in cells expressing DN-FGFR+GFP $(\mathrm{p}<0.019)$. Error bars are s.e.m. 


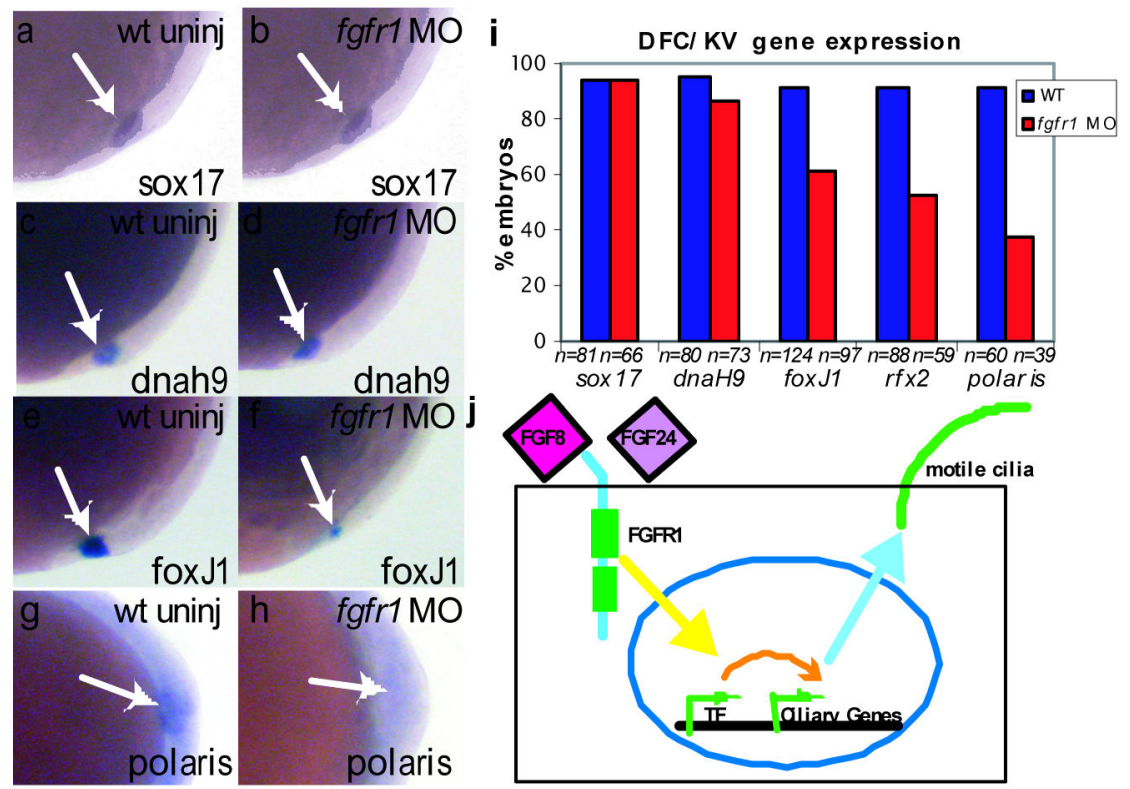

Figure 4. FGF signaling controls ciliogenic genes in DFC/KV cells $(\mathrm{a}, \mathrm{b})$ sox 17 expression in DFC/KV (and endoderm cells in a different focal plane) in $90 \%$ epiboly embryos was normal in $f g f r l$ morphants and WT embryos. (c, d) Expression of dnah9 in $95 \%$ epiboly embryos was normal in $f g f r l$ morphants and WT embryos. (e, f) In contrast, foxJ1 was down-regulated in $f g f r 1$ morphants versus WT embryos at $90 \%$ epiboly. $(\mathrm{g}, \mathrm{h})$ Similarly, polaris was down-regulated in $f g f r l$ morphants versus WT embryos at tailbud stage. (i) Comparison of percentage of embryos with WT expression levels of each gene indicated. (j) Proposed mechanism by which FGF signaling controls length of motile cilia: FGF ligands bind to FGFR1 activating downstream transcription factors (TF) including foxj 1 and $r f x 2$, these TF activate IFT genes (e.g. polaris) to maintain motile cilia length on epithelial cells. 